VOL. 61 (2000) [451-454]

\title{
ASPLUND SPACES AND A VARIANT OF WEAK UNIFORM ROTUNDITY
}

\author{
JOHN GILES AND JON VANDERWERFF
}

\begin{abstract}
We introduce a property formally weaker than weak uniform rotundity, which we call equatorial weak uniform rotundity. We show that an equatorially weakly uniformly rotund norm need not be weakly locally uniformly rotund. Nevertheless, we show that an equatorially weakly uniformly rotund Banach space is an Asplund space.
\end{abstract}

In [2], Borwein, Moors and Wang proved the striking result that, in the category sense, almost all 1-Lipschitz functions on a Banach space have maximal Clarke subdifferential. While this illustrates certain limitations of the Clarke derivative which has numerous applications in optimisation (see [3]), on the other hand, it affirms that each Banach space has a rich supply of Lipschitz functions. In [1], study was made of those Banach spaces where any bounded 1-Lipschitz function on any closed proper subspace can be extended to a 1-Lipschitz function on the whole space with maximal Clarke subdifferential. This property was shown to be connected to the following separation property for Lipschitz functions which ensures a sufficiently rich class of 1-Lipschitz functions extending any given bounded 1-Lipschitz function. A Banach space $X$ is Lipschitz separated if given any bounded 1-Lipschitz function $f$ on any closed proper subspace $Y$ of $X$ and $\bar{x} \notin Y$ one has $\sup \tilde{f}(\bar{x})>\inf \tilde{f}(\bar{x})$ where the supremum and infimum are over all 1-Lipschitz functions $\tilde{f}$ extending $f$ to $X$.

In this note, we work in real Banach spaces $X$ with closed unit ball $B_{X}$ and unit sphere $S_{X}$. It was shown in [1, Theorem 3.2] that a Banach space $X$ is Lipschitz separated if and only if for every pair of sequences $\left\{x_{n}\right\},\left\{y_{n}\right\}$ in $B_{X}$ such that $\lim _{n, m \rightarrow \infty}\left\|x_{n}+y_{m}\right\|=2$, there is no $\phi \in X^{*}$ such that $\limsup _{n \rightarrow \infty} \phi\left(x_{n}\right)<0<\liminf _{n \rightarrow \infty} \phi\left(y_{n}\right)$. The double indexing in the preceding characterisation makes it a rather awkward form of rotundity. Thus we say that a Banach space is equatorially weakly uniformly rotund (EWUR) if given sequences $\left\{x_{n}\right\},\left\{y_{n}\right\}$ in $B_{X}$ with $\lim _{n \rightarrow \infty}\left\|x_{n}+y_{n}\right\|=2$ there is no $\phi \in X^{*}$ such that $\limsup _{n \rightarrow \infty} \phi\left(x_{n}\right)<0<\liminf _{n \rightarrow \infty} \phi\left(y_{n}\right)$ (this is properly stronger than the Lipschitz separated property by [1, Theorem 4.1]). Our terminology was motivated

Received 23rd August, 1999

Copyright Clearance Centre, Inc. Serial-fee code: 0004-9727/00 \$A2.00+0.00. 
by the definition that a Banach space is weakly uniformly rotund (WUR) [respectively weakly locally uniformly rotund (WLUR)] if $\left(x_{n}-y_{n}\right) \rightarrow_{w} 0$ whenever $x_{n}, y_{n} \in B_{X}$ and $\left\|x_{n}+y_{n}\right\| \rightarrow 2$ [respectively $x_{n} \rightarrow_{w} x$ whenever $x_{n}, x \in B_{X}$ and $\left\|x_{n}+x\right\| \rightarrow 2$ ].

Our first objective is to show that an EWUR norm is not necessarily WUR. In fact building on ideas of Smith [7, Example 2.9], we show that much more is true.

THEOREM 1. A separable Banach space with separable dual can be equivalently renormed to be EWUR and not WLUR.

Proof: Because $X^{*}$ is separable, $X$ admits an equivalent WUR norm $[4$, Corollary II.6.9(ii)] which we denote by $\|\cdot\|$. Consider a closed hyperplane $Y$ in $X$ and $e \notin Y$ so that each $x \in X$ can be written $x=t e+y$ where $t \in \mathbb{R}$ and $y \in Y$, and we define the equivalent norm $\|\cdot\|_{S}$ on $X$ by $\|x\|_{S}=|t|+\|y\|$. The norm advertised in the theorem is then defined by

$$
\|x\|=\left(\|x\|_{S}^{2}+\sum_{i=1}^{\infty} 2^{-i} f_{i}^{2}(y)\right)^{1 / 2},
$$

where $\left\{f_{i}\right\}_{i=1}^{\infty}$ is dense in $S_{Y^{*}}$. Observe that $\|\cdot\| \|$ is not WLUR, because $\|e\| \|=1$ and $\left\|y_{n}\right\| \rightarrow 1$ where $y_{n} \in S_{Y}$ is chosen so that $y_{n} \rightarrow_{w} 0$. ( $Y$ is not Schur because $Y^{*}$ is separable.) Now $\left\|e+y_{n}\right\| \rightarrow 2$ and so $\|\cdot\| \cdot \|$ is not WLUR.

To see that $\|\cdot\|$ is EWUR, suppose that $\left\|x_{n}\right\|,\left\|y_{n}\right\| \leqslant 1$ and $\left\|x_{n}+y_{n}\right\| \rightarrow 2$. Let us write $x_{n}=s_{n} e+u_{n}$ and $y_{n}=t_{n} e+v_{n}$ where $s_{n}, t_{n} \in \mathbb{R}$ and $u_{n}, v_{n} \in Y$. By passing to a subsequence we may assume $s_{n} \rightarrow s, t_{n} \rightarrow t, u_{n} \rightarrow_{w^{*}} u \in Y^{* *}$, $\left\|u_{n}\right\| \rightarrow a, v_{n} \rightarrow w^{*} v \in Y^{* *}$ and $\left\|v_{n}\right\| \rightarrow b$. (We used the fact that $B_{Y^{* *}}$ is $w^{*}$ sequentially compact because $Y^{*}$ is separable.) The definition of $\|\cdot\|$ implies that

$$
\lim _{n \rightarrow \infty}\left[2\left\|x_{n}\right\|_{S}^{2}+2\left\|y_{n}\right\|_{S}^{2}-\left\|x_{n}+y_{n}\right\|_{S}^{2}\right]=0 \text { and thus: }
$$

$$
\lim _{n \rightarrow \infty}\left[\left\|x_{n}\right\|_{S}+\left\|y_{n}\right\|_{S}-\left\|x_{n}+y_{n}\right\|_{S}\right]=0 \quad \text { and }
$$

$$
\begin{array}{r}
\lim _{n \rightarrow \infty}\left[\left\|x_{n}\right\|_{S}-\left\|y_{n}\right\|_{S}\right]=0 \\
\lim _{n \rightarrow \infty}\left[\left|s_{n}\right|+\left|t_{n}\right|-\left|s_{n}+t_{n}\right|\right]=0 \\
\lim _{n \rightarrow \infty}\left[\left\|u_{n}\right\|+\left\|v_{n}\right\|-\left\|u_{n}+v_{n}\right\|\right]=0 .
\end{array}
$$

Now (2) implies that $|s|+|t|=|s+t|$, and hence $s t \geqslant 0$. Because $\left\{f_{n}\right\}_{n=1}^{\infty}$ is dense in $S_{Y^{*}}$, the definition of $\left\|\left|\|\mid\|\right.\right.$ implies that $\left(u_{n}-v_{n}\right) \rightarrow_{w} 0$ and so $u=v$. If $s=t$, then $\left(x_{n}-y_{n}\right) \rightarrow_{w} 0$ and there is nothing further to do.

In the remaining case that $s \neq t$ (and hence $|s| \neq|t|$ because $s t \geqslant 0$ ), we show that

$$
u_{n} \rightarrow_{w} 0 \text { and } v_{n} \rightarrow_{w} 0
$$


Because $|s| \neq|t|$, (1b) implies that $a \neq b$. If $a=0$ or $b=0$, then (4) is true. If $a \neq 0$ and $b \neq 0$, we let $\widetilde{u}_{n}=u_{n} / a$ and $\widetilde{v}_{n}=v_{n} / b$. Then $\left\|\widetilde{u}_{n}\right\| \rightarrow 1$ and $\left\|\widetilde{v}_{n}\right\| \rightarrow 1$; moreover (3) and the triangle inequality imply $\left\|\tilde{u}_{n}+\tilde{v}_{n}\right\| \rightarrow 2$. Because $\|\cdot\|$ is WUR, $\left(\widetilde{u}_{n}-\widetilde{v}_{n}\right) \rightarrow_{w} 0$. Therefore, $u=0$ and (4) is true when $s \neq t$. In this case, for any $\phi \in X^{*}, \phi\left(x_{n}\right) \rightarrow \phi(s e)$ and $\phi\left(y_{n}\right) \rightarrow \phi(t e)$. Because st $\geqslant 0$, it follows that $\liminf _{n \rightarrow \infty} \phi\left(y_{n}\right)$ and $\limsup _{n \rightarrow \infty} \phi\left(x_{n}\right)$ have the same sign. Therefore $\|\cdot \mid\|$ is EWUR.

Thus EWUR norms are strictly weaker than WUR norms, and so EWUR spaces form a broader class of Lipschitz separated spaces than the WUR spaces. Further evidence of the signficance of EWUR spaces is presented in the following extension of Hájek's important result [6, Theorem 1] asserting that a WUR Banach space is an Asplund space.

Theorem 2. An EWUR Banach space is an Asplund space.

Proof: First recall that a Banach space is an Asplund space if and only if every separable subspace has a separable dual (see [4, Theorem 1.5.7]). Because EWUR norms are inherited by subspaces, it is sufficient to work with a separable Banach space $X$. We suppose that $X^{*}$ is nonseparable and proceed by contraposition. Fix $\varepsilon>0$. Let $Y$ be a proper closed nonseparable subspace of $X^{*}$. Choose $y^{*} \in B_{X^{*}}$ such that $d\left(y^{*}, Y\right)>1 / 2$. Then there is an $F \in B_{X^{* *}}$ such that $F(Y)=\{0\}$ and $F\left(y^{*}\right)>1 / 2$. Given that $\omega_{1}$ is the first uncountable ordinal, one can use transfinite induction and the separation theorem to construct $\left\{f_{\alpha}\right\}_{\alpha<\omega_{1}} \subset Y$ and $\left\{G_{\alpha}\right\}_{\alpha<\omega_{1}} \subset X^{* *}$ such that $\left\|f_{\alpha}\right\|=1$ and $\left\|G_{\alpha}\right\|<1+\varepsilon / 2$ for all $\alpha$, and moreover $G_{\beta}\left(f_{\alpha}\right)=0$ if $\alpha<\beta$, $G_{\alpha}\left(f_{\alpha}\right)=1$, and $G_{\alpha}\left(y^{*}\right) \geqslant 0$ for all $\alpha$. Now let $F_{\alpha}=G_{\alpha}+(\varepsilon / 2) F$.

Then the system $\left\{f_{\alpha}, F_{\alpha}\right\}_{\alpha<\omega_{1}}$ satisfies the properties of the like-named system in the proof of [5, Lemma 3, p.234], and it moreover satisfies $F_{\alpha}\left(y^{*}\right)>\varepsilon / 4$ for all $\alpha<\omega_{1}$. Using this system in the proof of [5, Lemma 3, p.234] allows one to choose the $x_{n}$ 's in [5, Lemma 3, p.234] with the additional property that $y^{*}\left(x_{n}\right)>\varepsilon / 4$ for all $n$ (this is done with Helly's theorem and the additional condition $F_{\alpha}\left(y^{*}\right)>\varepsilon / 4$ for all $\alpha$ ). Therefore, we may assume that the $x_{n}$ 's in the Haar system of [5, Lemma 4, p.239] also satisfy $y^{*}\left(x_{n}\right)>\varepsilon / 4$.

Now use the above modified Haar system in the proof of [6, Theorem 1]. The $x_{t}^{\prime} s$ indexed on the binary tree thus satisfy $y^{*}\left(x_{t}\right)>\varepsilon / 4$ for all $t$. The proof of [6, Theorem 1] shows that there are $x_{n}, y_{n} \in X$ and $f_{b} \in X^{*}$ satisfying $2\left\|x_{n}\right\|^{2}+2\left\|y_{n}\right\|^{2}-$ $\left\|x_{n}+y_{n}\right\|^{2} \rightarrow 0$ while

$$
\lim _{n \rightarrow \infty} f_{b}\left(x_{n}\right)=1 \text { and } \lim _{n \rightarrow \infty} f_{b}\left(y_{n}\right)=0
$$

Now, note that [6, Claim 2, p.243] could have asserted that $x_{b}=\sum_{t_{1}<t<t_{b}} \alpha_{t} x_{t}$ and 
$y_{b}=\sum_{t_{b}<t<r_{b}} \beta_{t} x_{t}$ where $\sum_{t_{1}<t<t_{b}} \alpha_{t}=\sum_{t_{b}<t<r_{b}} \beta_{t}=1$ and $\alpha_{t} \geqslant 0$ and $\beta_{t} \geqslant 0$ rather than allowing $\left|\alpha_{t}\right| \leqslant 1$ and $\left|\beta_{t}\right| \leqslant 1$. Because of this, we may assume that each $y_{n}$ is a convex combination of some $x_{t}$ 's. Therefore,

$$
y^{*}\left(y_{n}\right)>\varepsilon / 4 \text { for all } n .
$$

By passing to an appropriate subnet, we let $\bar{x}$ be the $w^{*}$ limit of $\left\{x_{n_{\lambda}}\right\}$ and let $\bar{y}$ be the $w^{*}$ limit of $\left\{y_{n_{\lambda}}\right\}$. Now, (5) implies that $f_{b}(\bar{x}+\bar{y})=1$ and $f_{b}(\bar{y})=0$, thus if $\bar{y}$ were on the line through the origin and $\bar{x}+\bar{y}$, the only possibility would be $\bar{y}=0$. However, (6) rules out that possibility, and so $\bar{y}$ is not on the line through $(\bar{x}+\bar{y}) / 2$ and the origin. According to the separation theorem, there is $\phi \in X^{*}$ with $\phi(\bar{y})>0>\phi(\bar{x})$. Therefore $\lim \sup _{\lambda} \phi\left(x_{n_{\lambda}}\right)<0<\liminf \operatorname{in}_{\lambda} \phi\left(y_{n_{\lambda}}\right)$. By extracting an appropriate subsequence from this net with $\left\|y_{n_{\lambda_{k}}}+x_{n_{\lambda_{k}}}\right\| \rightarrow 2$, we deduce that $X$ is not EWUR.

In summary, $X$ is WUR $\Rightarrow X$ is EWUR $\Rightarrow X$ is Lipschitz separated; also $X$ is EWUR $\Rightarrow X$ is Asplund, and none of the arrows reverse (see Theorem 2, and [1, Theorem 4.1, Example 3.6b]). The following natural question remains open.

QUESTION. Is a Lipschitz separated Banach space an Asplund space?

\section{REFERENCES}

[1] J.M. Borwein, J.R. Giles and J. Vanderwerff, 'Rotund norms, Clarke subdifferentials and extensions of Lipschitz functions', (CECM Preprint 98:126).

[2] J.M. Borwein, W.B. Moors and X. Wang, 'Generalized subdifferentials: a Baire categorical approach', (CECM Preprint 99:127).

[3] F.H. Clarke, Optimization and nonsmooth analysis, Classics in Applied Mathematics 5 (SIAM, Philadelphia, PA, 1990).

[4] R. Deville, G. Godefroy and V. Zizler, Smoothness and renormings in Banach spaces, Pitman Monographs and Surveys in Pure and Applied Mathematics 64 (Longman, Harlow, U.K., 1993).

[5] J. Diestel, Geometry of Banach spaces-selected topics, Lecture Notes in Mathematics 485 (Springer-Verlag, Berlin, Heidelberg, New York, 1975).

[6] P. Hájek, 'Dual renormings of Banach spaces', Comment. Math. Univ. Carolin. 37 (1996), 241-253.

[7] M.A. Smith, 'Banach spaces that are uniformly rotund in weakly compact sets of directions', Canad. J. Math. 29 (1977), 963-970.

Department of Mathematics The University of Newcastle Newcastle NSW 2308 Australia
Department of Mathematics

La Sierra University

Riverside CA 92515

United States of America 